\title{
Dissipation strength of the tilting degree of freedom in fusion-fission reactions
}

\author{
P. N. Nadtochy, D. V. Vanin, A. V. Cheredov, S. V. Fedorov, \\ E. G. Ryabov and G. D. AdeEv
}

Omsk State University, Physics Department, Mira prospekt 55-A, Omsk, 644077, Russia

\begin{abstract}
The four-dimensional Langevin model was applied to calculate a wide set of experimental observables for compound nuclei, formed in heavy-ion fusion-fission reactions. A modified one-body mechanism for nuclear dissipation with a reduction coefficient $k_{\mathrm{s}}$ of the contribution from a "wall" formula was used for shapes parameters. Different possibilities of deformation-dependent dissipation coefficient for the $\mathrm{K}$ coordinate $\left(\gamma_{K}\right)$ were investigated. Presented results demonstrate that the influence of the $k_{\mathrm{S}}$ and $\gamma_{K}$ parameters on the calculated quantities can be selectively probed. It was found that it is possible to describe experimental data with the deformation-dependent $\gamma_{K}$ coefficient. One of the possibility is to use large values of $\gamma_{K} \simeq 0.2(\mathrm{MeV} \mathrm{zs})^{-1 / 2}$ for compact shapes featuring no neck and small values of $\gamma_{K} \simeq 0.0077$ $(\mathrm{MeV} z s)^{-1 / 2}$ for elongated shapes.
\end{abstract}

Fission still is one of the most interesting and challenging topics in nuclear physics providing a perfect opportunity to investigate the large scale evolution of initial compound nucleus into fission products. During the past two decades stochastic approach based on multidimensional Langevin equations has been extensively and rather successfully used to elucidate many problems of collective nuclear dynamics in fusion-fission reactions at high excitation energies $[1,2]$. A reasonable choice of collective degrees of freedom for modeling shape evolution and considering particle evaporation allow modeling the complex interplay between static and dynamical effects in fission and succeeding in explaining a wide range of experimental data $[3,4]$. 


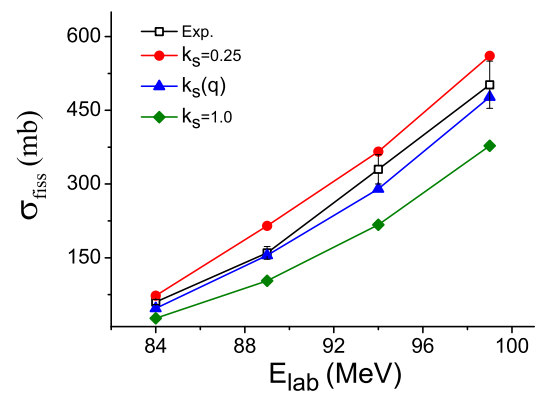

Figure 1: (Color online) The fusionfission cross section for ${ }^{204}$ Po. The open squares are experimental data. The filled symbols are results of $4 \mathrm{D}$ calculations: circles with $k_{s}=0.25$, triangles with $k_{s}(\mathbf{q})$, and diamonds $k_{s}=1$.

The significance of orientation degree of freedom ( $\mathrm{K}$ coordinate), which is the projection of the total angular momentum onto the symmetry axis of fissioning nucleus, was demonstrated and an overdamped Langevin equation for $\mathrm{K}$ coordinate were introduced $[5,6]$.

In the present study we used recently developed $4 \mathrm{D}$ dynamical model for the description of fissioning nucleus shape evolution based on a stochastic approach $[1,2,4,7,8]$. The detailed description of the model could be found in Refs. [2,4] and here we give only a short summary. Three collective shape coordinates and the orientation degree of freedom ( $\mathrm{K}$ coordinate) were considered dynamically from the ground state deformation to the scission into fission fragments. A modified one-body mechanism of nuclear dissipation $[9,10]$ was employed to determine the dissipative part of the driving forces with reduction coefficient from the "wall" formula $k_{s}$. The value $k_{s}=1.0$ corresponds to the "wall" and "wall-plus-window" formulas, whereas values $0.2<k_{s}<0.5$ allow to reproduce different features of the experimental fission fragment MED and particle multiplicities in multidimensional Langevin calculations $[2,8,11]$.

In Refs. [12-14] it was argued that chaos-theory related ideas [14] can be used to calculate the value of the reduction coefficient $k_{\mathrm{S}}$ as a function of deformation of the fissioning nucleus. The applications of this approach to calculate the coefficient $k_{\mathrm{S}}(\mathbf{q})$ for studying different fission characteristics were rather successful, and have shown that such calculations yield almost the same results as those using the constant $k_{\mathrm{S}}$ coefficient from the interval $0.25<k_{\mathrm{s}}<0.5[6,12,15]$.

We use three different options to model the deformation dependence of $\gamma_{K}(\mathbf{q})$ coefficient as described in Ref. [16]. As the first one we used 




Figure 2: (Color online) The anisotropy of fission fragment angular distribution at $k_{s}$ (q) (a) and $k_{s}=0.5$ (b) for ${ }^{204} \mathrm{Po}$. The open squares are experimental data. The filled triangles correspond to $4 \mathrm{D}$ calculations with $\gamma_{K}^{(1)}=0.077(\mathrm{MeV} \mathrm{zs})^{-1 / 2}$; the inverted triangles with $\gamma_{K}^{(2)}\left(\gamma_{K}^{\text {const }}=0.2\left(\mathrm{MeV} \mathrm{zs}^{-1 / 2}\right)\right.$; and the circles with $\gamma_{K}^{(3)}$.

$\gamma_{K}^{(1)}=$ const. The second option is determined by the following equation

$$
\gamma_{K}^{(2)}(\mathbf{q})=\left\{\begin{array}{l}
\gamma_{K}^{\text {const }}, \text { for shapes without a neck; } \\
\gamma_{K}^{\text {neck }}(\mathbf{q}), \text { for shapes with a neck }
\end{array}\right.
$$

where $\gamma_{K}^{\text {const }}$ is a variable parameter independent of nuclear deformation. After appearance of the neck in nuclear shape the $\gamma_{K}^{\text {const }}$ value is joined smoothly with $\gamma_{K}^{\text {neck }}(\mathbf{q})$, which was obtained in Refs. $[5,16]$. The third one is given by equation

$$
\gamma_{K}^{(3)}(\mathbf{q})=\left\{\begin{array}{l}
\gamma_{K}^{\text {cshape }}(\mathbf{q}), \text { for shapes without a neck } \\
\gamma_{K}^{\text {neck }}(\mathbf{q}), \text { for shapes with a neck }
\end{array}\right.
$$

where $\gamma_{K}^{\text {cshape }}(\mathbf{q})$ is the extrapolation of $\gamma_{K}^{\text {neck }}(\mathbf{q})$ to the mononuclear shapes, featuring no neck. The detailed description of these three prescriptions could be found in Ref. [16].

In the present calculations we investigate the influence of $k_{s}$ and deformation-dependent $\gamma_{K}(\mathbf{q})$ parameters on the fusion-fission $\left(\sigma_{f i s}\right)$, evaporation residues $\left(\sigma_{E R}\right)$ cross sections, and anisotropy of fission fragment angular distribution in 4D dynamical model. We performed calculations for the reactions ${ }^{16} \mathrm{O}+{ }^{188} \mathrm{Os} \rightarrow{ }^{204} \mathrm{Po}\left(E_{l a b}=84,89,94\right.$, and $\left.99 \mathrm{MeV}\right)[17-19]$ and ${ }^{16} \mathrm{O}+{ }^{184} \mathrm{Pt} \rightarrow{ }^{200} \mathrm{~Pb}\left(E_{l a b}=91.6,102.5\right.$, and $\left.107.9 \mathrm{MeV}\right)[20-23]$. The calculations were performed at different values of $k_{s}$ and $\gamma_{K}$. In figures we present the options which allow good reproduction of experimental data. In 
fig. 1 one can see that $k_{s}=0.25$ or $k_{s}(\mathbf{q})$ obtained from chaos-weighted wall formula allows well reproduce the fission cross section for the fission of ${ }^{204} \mathrm{Po}$. The calculated fission cross section does not depend neither on the $\gamma_{K}$ option used nor on the absolute $\gamma_{K}$ values, so we did not show it in fig. 1 in order not to complicate figure. Our investigations [16] show that only the anisotropy of fission fragment angular distribution is dependent on both $k_{s}$ and $\gamma_{K}$ values, as it was found in previous investigation with heavy compound nuclei [6]. The calculated anisotropy of fission fragment angular distribution is presented in fig 2 . One can see that at both $k_{s}$ values $\left(k_{s}=0.5\right.$ and $\left.k_{s}(\mathbf{q})\right)$ it is possible to reproduce the experimental data on anisotropy at constant $\gamma_{K}=0.077(\mathrm{MeV} \mathrm{zs})^{-1 / 2}$ or with the deformation-dependent $\gamma_{K}$ coefficient: to use large values of $\gamma_{K} \simeq 0.2(\mathrm{MeV} \mathrm{zs})^{-1 / 2}$ for compact shapes featuring no neck and small values of $\gamma_{K}(\mathbf{q})$ for dinuclear shapes. The $4 \mathrm{D}$ calculations with $\gamma_{K}^{(3)}(\mathbf{q})$ option underestimate the experimental anisotropy of fission fragment angular distribution.

The comparison of theoretical 4D calculations with experimental data for the ${ }^{200} \mathrm{~Pb}$ is presented in fig. 3. In figure 3(a) the calculated fusion-fission $\left(\sigma_{f i s}\right)$ and evaporation residues $\left(\sigma_{E R}\right)$ cross sections are compared with the experimental data at different excitation energies and $k_{s}$ values. We found that $\gamma_{K}$ does not influence the cross sections, so we did not present in fig. 3(a) the results obtained with different $\gamma_{K}$ values. One can see that $4 \mathrm{D}$ calculations reproduce worse the cross sections for the ${ }^{200} \mathrm{~Pb}$ compound nucleus than for the ${ }^{204} \mathrm{Po}$ compound nucleus. The same is true also for the calculated results for the anisotropy of fission fragment angular distribution presented in fig. 3(b). In the present calculations we needed to increase the $k_{s}$ and/or $\gamma_{K}$ values, which generate large values of the anisotropy. However, even very large values of $\gamma_{K}$ (the option $\gamma_{K}^{(2)}(\mathbf{q})$ with the large values of $\gamma_{K}^{\text {const }}=0.4-0.6(\mathrm{MeV} \mathrm{zs})^{-1 / 2}$ for compact shapes $)$ and large $k_{s}$ values does not allow to reproduce the experimental values of the anisotropy of fission fragment angular distribution. Moreover, the anisotropy reaches the saturation at $\gamma_{K}^{\text {const }}=0.4(\mathrm{MeV} \mathrm{zs})^{-1 / 2}$, and does not grow up after the further increase of $\gamma_{K}^{\text {const }}$ independently on $k_{s}$ value used. The results of present $4 \mathrm{D}$ calculations with $k_{s}=1.0$ and $\gamma_{K}^{(2)}(\mathbf{q})$ with the large values of $\gamma_{K}^{\text {const }}=0.4-0.6(\mathrm{MeV} \mathrm{zs})^{-1 / 2}$ for compact shapes predicts the anisotropy about $15 \%$ less than experimental data. However, we should mention that there is significant difference in experimental data on fusion cross section for the ${ }^{200} \mathrm{~Pb}$ compound nucleus between old [23] and recent [20] experimental data. Thus, possible revision of experimental data [23] on anisotropy of angular distribution could influence on the conclusions from comparison 

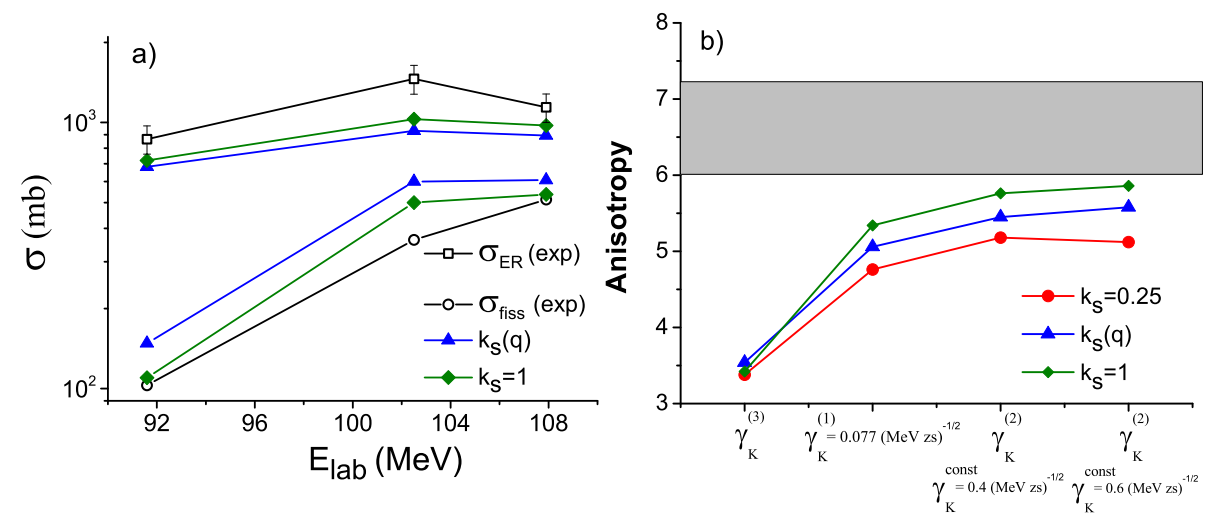

Figure 3: (Color online) The $\sigma_{f i s}$ and $\sigma_{E R}$ (a) and the anisotropy of fission fragment angular distribution (b) for ${ }^{200} \mathrm{~Pb}$ at different $k_{s}$ values and $\gamma_{K}$ options. The panel (b) demonstrates results for the $E_{l a b}=107.9 \mathrm{MeV}$. The open symbols are experimental data. The hatched area at panel (b) determines experimental data with error bars. The filled symbols are results of $4 \mathrm{D}$ calculations: circles with $k_{s}=0.25$; triangles with $k_{s}(\mathbf{q})$; and diamonds with $k_{s}=1.0$.

between theoretical and experimental data.

Concluding we can state that it was found that in the 4D Langevin calculations it is possible to describe experimental data with the deformationdependent $\gamma_{K}$ coefficient. One of the possibility is to use large values of $\gamma_{K} \simeq 0.2(\mathrm{MeV} \mathrm{zs})^{-1 / 2}$ for compact shapes featuring no neck and small values of $\gamma_{K} \simeq 0.0077(\mathrm{MeV} \mathrm{zs})^{-1 / 2}$ for elongated shapes. Using such a different value of $\gamma_{K}$ for deformations before saddle and along the saddleto-scission descent tends the dynamics of the $\mathrm{K}$ coordinate to the prediction of the transition state model at saddle point.

This study was partially supported by the Russian Foundation for Basic Research, Research Project No. 13-02-00168 (Russia).

\section{References}

[1] Abe Y., Ayik S., Reinhard P-G., Suraud E., Phys. Rep., 275 (1996) 49.

[2] Adeev G. D.et al., Fiz. Elem. Chast. At. Yadra, 36 (2005) 732.

[3] Vardaci E. et al., Phys. Rev. C, 92 (2015) 034610.

[4] Nadtochy P. N., Ryabov E. G., Gegechkori A. E., Anischenko Yu. A., Adeev G. D., Phys. Rev. C, 85 (2012) 064619. 
[5] Lestone J. P. and McCalla S. G., Phys. Rev. C, 79 (2009) 044611.

[6] Nadtochy P. N., Ryabov E. G., Gegechkori A. E., Anischenko Yu. A., Adeev G. D., Phys. Rev. C, 89 (2014) 014616.

[7] Kramers H. A., Physica, 7 (1940) 284.

[8] Nadtochy P. N., Adeev G. D., Karpov A. V., Phys. Rev. C, 65 (2002) 064615.

[9] Randrup J. and Swiatecki W. J., Nucl. Phys. A, 429 (1984) 105.

[10] Nix J. R. and Sierk A. J., in Proceedings of the 6th Adriatic Conference on Nuclear Physics: Frontiers of Heavy Ion Physics, Dubrovnik, Yugoslavia, 1987 ed N. Cindro, R. Caplar, W. Greiner (World Sci., Singapore) 1990, pp. 333-340.

[11] Ryabov E. G., Karpov A. V., Nadtochy P. N., Adeev G. D., Phys. Rev. $C, \mathbf{7 8}(2008) 044614$.

[12] Pal S. and Mukhopadhyay T., Phys. Rev. C, 54 (1996) 1333.

[13] Blocki J., Brut F., Srokowski T., Swiatecki W. J., Nucl. Phys. A, 545 (1992) 511.

[14] Swiatecki W. J., Nucl. Phys. A, 488 (1988) 375c.

[15] Schmitt C., Mazurek K., Nadtochy P. N., Phys. Lett. B, 737 (2014) 289.

[16] Nadtochy P. N., Ryabov E. G., Adeev G. D., J. Phys. G, 42 (2015) 045107.

[17] J. van der Plicht et al., Phys. Rev. C, 28 (1983) 2022.

[18] Rafiei R. et al., Phys. Rev. C, 77 (2008) 024606.

[19] Tripathi R. et al., Phys. Rev. C, 79 (2009) 064607.

[20] Shidling P. D. et al., Phys. Rev. C, 74 (2006) 064603.

[21] Leigh J. R., Bokhorst J. J. M., Hinde D. J., Newton J. O., J. Phys. G, 14 (2015) L55.

[22] Hinde D. J. et al., Nucl. Phys. A, 385 (1982) 109.

[23] Forster J. S. et al., Nucl. Phys. A, 464 (1987) 497. 